\title{
An Online Robot Collision Detection and Identification Scheme by Supervised Learning and Bayesian Decision Theory
}

\author{
Zengjie Zhang, Student Member IEEE, Kun Qian*, Member IEEE, \\ Björn Schuller, Fellow IEEE and Dirk Wollherr, Senior Member IEEE
}

\begin{abstract}
This paper is dedicated to developing an online collision detection and identification scheme for human-collaborative robots. The scheme is composed of a signal classifier and an online diagnosor, which monitors the sensory signals of the robot system, detects the occurrence of a physical human-robot interaction and identifies its type within a short period. In the beginning, we conduct an experiment to construct a data set that contains the segmented physical interaction signals with ground truth. Then, we develop the signal classifier on the data set with the paradigm of supervised learning. To adapt the classifier to the online application with requirements on response time, an auxiliary online diagnosor is designed using Bayesian decision theory. The diagnosor provides not only a collision identification result but also a confidence index which represents the reliability of the result. Compared to the previous works, the proposed scheme ensures rapid and accurate collision detection and identification even in the early stage of a physical interaction. As a result, safety mechanisms can be triggered before further injuries are caused, which is quite valuable and important towards a safe humanrobot collaboration. In the end, the proposed scheme is validated on a robot manipulator and applied to a demonstration task with collision reaction strategies. The experimental results reveal that the collisions are detected and classified within $20 \mathrm{~ms}$ with an overall accuracy of $99.6 \%$, which confirms the applicability of the scheme to collaborative robots in practice.
\end{abstract}

Note to Practitioners-This paper is intended to provide a novel online collision event handling scheme for robots in industrial environments. This scheme is designed to quickly and accurately detect an accidental collision and distinguish it from the intentional human-robot interaction. The method takes the raw signals from external torque sensors and provides a collision diagnosis result with a reliability index. The simple structure makes it easy to be implemented as a regular fault monitoring routine for collaborative robots. Different from the conventional methods, the

* Corresponding author.

This work was partially supported by the Horizon 2020 program under grant 820742 of the project "HR-Recycler", the China Scholarship Council under Grant 201506120029, the Zhejiang Lab's International Talent Fund for Young Professionals (Project HANAMI), P.R. China, the JSPS Postdoctoral Fellowship for Research in Japan (ID No. P19081) from the Japan Society for the Promotion of Science (JSPS), Japan, and the Grants-in-Aid for Scientific Research (No. 19F19081 and No. 17H00878) from the Ministry of Education, Culture, Sports, Science and Technology (MEXT), Japan.

Z. Zhang and D. Wollherr are with the Chair of Automatic Control Engineering, Technical University of Munich, Theresienstr. 90, 80333, Munich, Germany (e-mail: \{zengjie.zhang, dw $@ @$ tum.de).

K. Qian is with the Educational Physiology Laboratory, Graduate School of Education, The University of Tokyo, 7-3-1 Hongo, Bunkyo-ku, 113-0033 Tokyo, Japan (e-mail: qian@p.u-tokyo.ac.jp).

B. Schuller is with the GLAM - Group on Language, Audio \& Music, Imperial College London, 180 Queens' Gate, Huxley Bldg., London SW7 2AZ, UK, and also with the ZD. B Chair of Embedded Intelligence for Health Care and Wellbeing, Universität Augsburg, Eichleitnerstr. 30, 86159 Augsburg, Germany (email: schuller@ieee.org). proposed collision identification scheme in this paper especially focuses on overcoming the following two challenges in practice: firstly, to timely and accurately report a collision within its early stage; secondly, to ensure a high identification accuracy in a complicated environment, where ubiquitous disturbance and noise are unneglectable. The experimental validation at the end of this paper confirms its promising application value in humanrobot collaboration.

Index Terms - collision detection and identification, anomaly monitoring, human-robot interaction, fault detection and isolation, robot safety, supervised learning, collision event pipeline.

\section{INTRODUCTION}

$\mathrm{S}$ AFETY is always a critical issue for human-robot collaborative tasks, especially when physical human-robot interaction (pHRI) is demanded [1], [2]. Different types of pHRI exert various impacts on humans. The intentional contacts desired by the cooperative tasks, such as the human teaching processes, are usually quite safe. On the contrary, the accidental collisions, which may lead to unexpected injuries or damages, are often dangerous to humans. As pointed out by many surveys on the safety of human-robot collaboration [1], [3], the accidental collisions are inevitable in pHRI and should be carefully handled. Especially, the reaction strategy for the accidental collisions should be distinguished from the intentional contacts since they tend to cause opposite consequences, which motivates the research on collision event handling. In the literature, a typical collision event handling pipeline usually contains two procedures, namely the precollision and the post-collision ones [4], [5]. The development of such a pipeline involves various topics, including collision avoidance [6], [7], collision force estimation [8], [9], collision detection and identification (CDI) [10], [11] and collision reaction strategy design [12], [13]. These topics are corresponding to different components in the pipeline.

This paper is specifically concerned with the CDI problem of robot systems. As part of the post-collision procedure, it is intended to monitor the sensory signals, detect the occurrence of pHRI and identify whether it is an accidental collision or an intentional contact. The results of CDI will be given to the collision reaction procedures, where the corresponding safe strategy is enabled to avoid further harms [5]. As claimed in [5], accidental collisions and intentional contacts possess distinguishable properties, which are reflected in the features of the sensory signals. Considering this, one can formulate 
robot CDI as a multi-class classification problem, with collision, contact, and free respectively denoting the accidental collisions, intentional contacts and the nominal cases where no pHRI occurs. Then, the main target of CDI becomes to develop a pHRI signal classifier using a data set that contains the pHRI waveform. In general, this proposes a classification problem for time series.

For such a problem, expertise can be found in related work, which will be discussed in detail in Sec. II. Generally, an important technique in the classification problem for time series is the segmentation of raw signals, since classifiers can not process series with infinite length. In a conventional paradigm of classifier development, the raw signals are segmented before predictions are conducted, even for the online applications. In the context of CDI, the conventional solutions typically design some detection logic, such as a signal threshold, to approximately mark the starting instant of a pHRI, and segment the online signals after observing the entire pHRI waveform, before producing a prediction result using a trained classier. As a result, an accurate prediction result can only be produced when the pHRI almost vanishes. By almost we mean that the pHRI waveform is contained in the signal segments as much as a correct identification result can be produced. Such a method, however, usually leads to a useless result in the context of safe human-robot collaboration, since a collision or a contact has already occurred and negative consequences may have already been caused. Intuitively speaking, a prediction result of the classifier does not really predict the occurrence of a collision. The reason for this issue is that the signal values in the future are not available for the segment at the current time, due to the causality of online time-series.

Nevertheless, this is a significant issue for safe human-robot collaboration, since A CDI scheme that can be practically applied is expected to accurately report the pHRI in its early stage, definitely before it vanishes, such that potential injuries can be prevented in advance. Unfortunately, such a valuable topic has attracted very little attention, probably due to the following two challenges. Firstly, accurate prediction results should be produced even when the segmented pHRI waveform is incomplete. From a general perspective, this introduces inconsistency between the distribution of the training data and that of the predicted samples, if the classifier is developed on the signal segments with complete pHRI waveform. This indicates that the identification result in the early stage of a pHRI is very likely to be incorrect. Secondly, an accidental collision is usually referred to as an instantaneous anomaly [14], which occurs unexpectedly and only lasts for a short period of time. Thus, it is more difficult to be identified than the other anomalies that are featured with low bandwidth and steady changes [15], [16]. The main target of this paper is to fill these gaps by proposing a novel online CDI scheme that ensures both high accuracy and fast response.

The remaining content of this paper is organized as follows. Sec. II summarizes the related work and clarifies the main contributions of this paper. Sec. III introduces the overall structure of the scheme and the collection process of the pHRI data set. The development of two important components of the scheme, the signal classifier, and the online diagnosor, is respectively presented in Sec. IV and Sec. V. In section VI, the proposed CDI scheme is validated on a seven-degree-offreedom (DoF) KUKA robot. The decent performance of the CDI scheme revealed by the experimental validation confirms its applicability in practice. Finally, section VII concludes the paper.

\section{RELATED WORK}

Conventionally, CDI for robot systems has been recognized as an engineering-oriented problem and is mainly solved by simple threshold logic or hypothesis testing based methods [11], [17], where the filter-based techniques are widely applied [18]. More recent work tends to formulate CDI as a classification problem for segmented time series. Similar work can be found in relevant fields, such as speech recognition [19], snoring identification [20], bird sound recognition [21] and fault identification of mechatronic systems [22]-[24]. The common ground of these applications is that the segmented time series, either acoustic signals or sensory streams, are expected to be classified as a certain type. In [25], the classification of time series is generally investigated by applying various data sets. In [26], a deep neural network is constructed to detect the system faulty status. In [27], various classifiers are developed and tested using the recorded signal samples. Similar work also includes [28], [29], where neural network is constructed to monitor the grasping slippages and colliding torques, and [30], [31], where SVM classifiers are developed to detect external collisions. Based on the mentioned work, a mature development framework for time series has been well-formed. However, such a framework is confronted with challenges when applied to CDI for robot systems with pHRI tasks, since the prediction results are only available after the collision vanishes or the entire pHRI waveform is segmented, which has been explained in Sec. I. Therefore, these methods are not suitable for human-robot collaboration with high requirements on human safety.

As alternatives, probabilistic series-models, such as the Hidden Markov Model (HMM) [32] and the Gaussian Mixture Model (GMM) [33] are also applied to CDI by exploiting the dependence properties of time series. In [34], an HMM is developed to detect exceptional events in an object-alignment robot task, where the measured torques and their derivatives are used. The work in [35] also develops an HMM model to realize a fault detection scheme for a feeding-assistant robot using multi-sensor signals. In [36], probabilistic support vector machine is used to detect online anomalies. Nevertheless, these series models require artificially assigned prior knowledge on the distributional dependence of the raw signals. In most practical scenarios, such knowledge is challenging to acquire, especially in a complicated environment where the distribution of the disturbance and noise is not treated as Gaussian.

The main contribution of this paper is to develop an online CDI scheme for safe human-robot collaboration. The major advantage of this novel scheme over the conventional methods is to produce fast and accurate CDI in the early stage of pHRI. Apart from the identification result, it also provides a confidence index to indicate how much the result can be 
trusted, which benefits the design of a flexible collision handling pipeline in future work. Moreover, the scheme does not require extra detection logic and any prior knowledge on the dependence of pHRI signals. Therefore, no heuristic thresholds or distribution assumptions are needed. Another contribution of this paper is to provide a pHRI data set with manual labels specifically for the CDI usage, which is still lacking in the literature. Different from most of the previous work where the torque sensors are installed on the end-effectors, the signal data we used in this paper is from the torque sensors on the robot joints, which can even detect pHRIs on robot links. On the other hand, the data set contains more interfering signals due to coupled mechanical vibrations and strong current noise on the sensors, which leads to a larger challenge to the development of the CDI scheme. From another perspective, however, our work reflects the complicated conditions in practice, which provides an outcome truly with application values.

\section{The Scheme Overview And DAta Collection}

In this section, we introduce the overall structure of the proposed CDI scheme. To develop this scheme, we conducted a data collection experiment with manual collisions and contacts to construct a pHRI data set. The data comes from the segmentation of the external torques that are measured by shaft-mounted torque sensors installed on the robot joints.

\section{A. Overview of the CDI Scheme}

The general structure of the proposed online CDI scheme and the development flowchart of each component are illustrated in Fig. 1. During an online execution, the CDI routine takes the torque signal $\tau$ and segment it recurrently. Then, the features extracted from the signal segment $\mathcal{T}$ are given to the classifier which produces a prediction result $r$ among collision, contact and free. Based on a series of prediction results, the online diagnosor offers a CDI diagnosis result $C^{*}$ and a confidence index $\mathcal{I}$. In this framework, no heuristic threshold values are needed to mark the occurrence time of the pHRI.

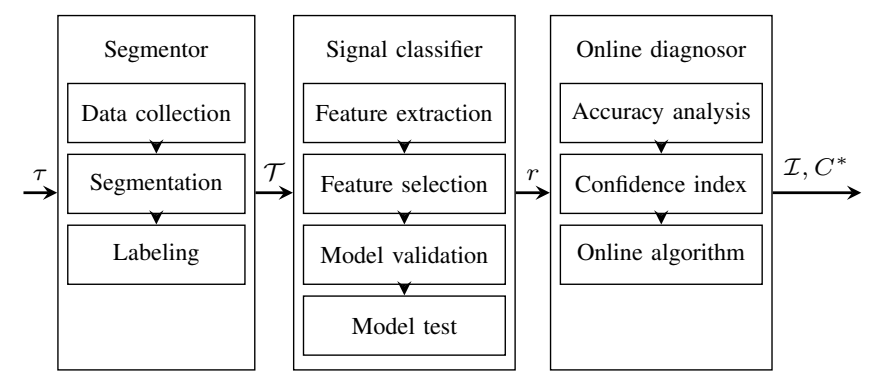

Fig. 1. The structural overview of the proposed online CDI scheme and the corresponding development flowcharts, where $\tau$ is the measured torque signal, $\mathcal{T}$ is the signal segment, $r$ is the prediction result of the signal classifier, and $C^{*}, \mathcal{I}$ are respectively the ultimate diagnosis result and its confidence index.

\section{B. Data Collection}

To construct the pHRI data set for the development of the signal classifier, we conduct a collection experiment using a seven DoF KUKA LWR4+ robot manipulator [37], which is firmly mounted onto a fixed platform. During the experiment, different types of physical interaction in various strengths and directions is manually exerted on the robot end-effector by seven subjects hired for this experiment, aiming to include individual uncertainties in the collected data, so as to ensure the generalization ability of the data set. In the experiment, accidental collisions are created using a soft hammer with fast hand speed and tough strength, whilst intentional contacts are made by gloved hands with compliant forces. A spherical plastic end-effector is specially designed to bear the contact forces from arbitrary directions.

Note that the pHRI is exerted during the movement of the robot, such that the measured signals contain drifts and noise caused by the joint motions. It is worth mentioning that the drifts and noise are also affected by the direction of the joint motion. Considering this, we apply several different robot trajectories that are assigned with the same starting position but different ending positions, such that both positive and negative motion directions are covered for each joint. The trajectories are designed in the joint space and interpolated by sinusoidal functions to ensure smooth motion. All these experiment configurations are intended to ensure a representative data distribution, which is important to ensure a high generalization ability to the classifier.

The motion of the robot is controlled by a trajectory tracking program that implements a continuous reciprocating motion of the manipulator between the starting position and each ending position. The program also reads the measured torque signals and records them as seven-dimensional time-series with a sampling period of $1 \mathrm{~ms}$. During the motion of the robot, one experimenter exerts an accidental collision or an intentional contact on the end-effector. At the same time, the other experimenter notes down the occurrence instant and the type of the pHRI. To obtain a balanced data set between collisions and contacts, we try to produce an equal amount of samples for these two classes.

\section{Signal Segmentation and Sample Labeling}

After collecting the raw pHRI signals, we conduct segmentation to split the signals into segments with fixed-length. Let us denote the width of the segments as $l$ and the bias as $b$, with the unit of ms. By bias we mean the time period between the occurrence instant of a pHRI and the ending instant of the segment, where we have $0 \leqslant b \leqslant l$. In general, $l$ determines how much signal information is included in the segments, whilst $b$ adjusts the proportion of the pHRI waveform in the segment. If the ending instant of the segment represents the current time, $b$ denotes the period after the occurrence of the corresponding pHRI. Therefore, the segmentation scheme is well determined by the two parameters $l$ and $b$. For the data set, the segment bias is typically set as $b=l$, since the torque values before the pHRIs are irrelevant to the classification. The determination of segment width $l$ is mainly based on the engineering experience. Usually, it should be large enough to contain sufficient information of the pHRI waveform. On the other hand, an overlarge width may involve irrelevant signals and lead to poor generalization ability. 


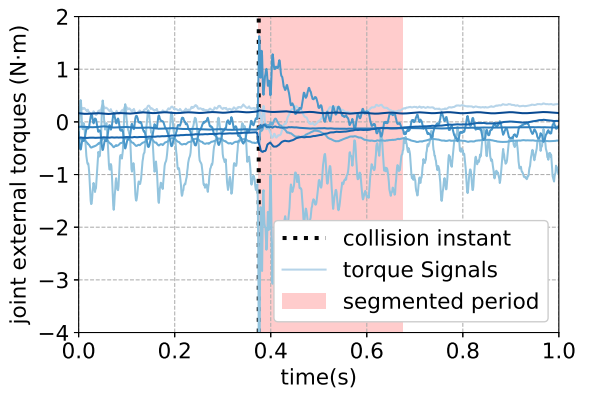

(a) Waveform of collision

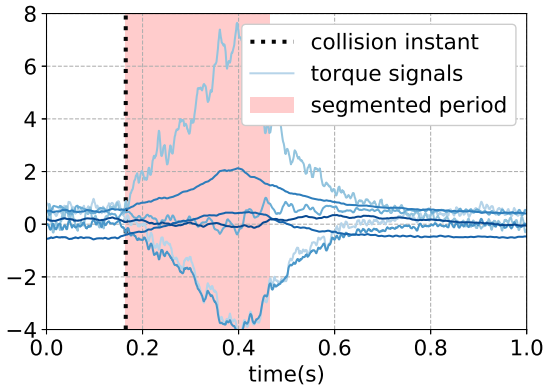

(b) Waveform of contact

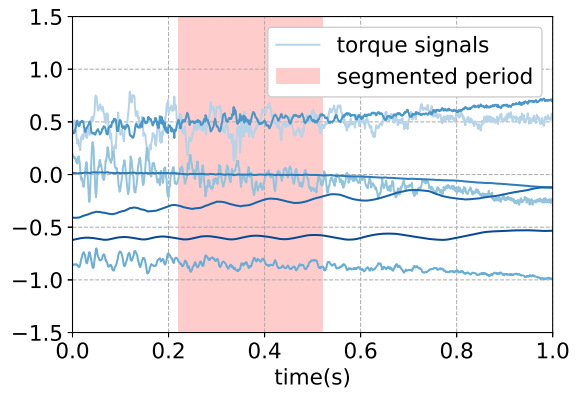

(c) Waveform of free

Fig. 2. The waveform of signals (external toques on 7 joints) respectively with classes of collision 2(a), contact 2(b) and free 2(c). The segmented partitions are marked with colored areas, where $b=l=300(\mathrm{~ms})$.

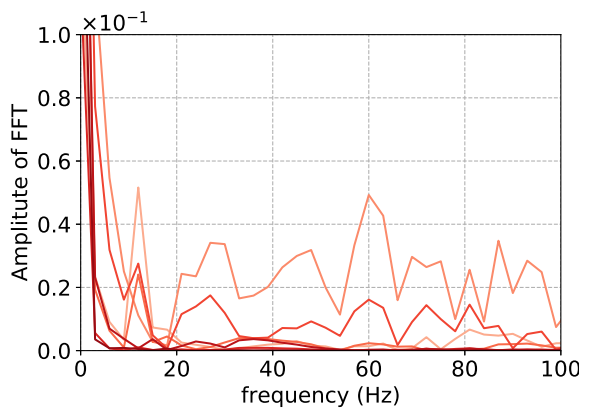

(a) Spectrum of collision

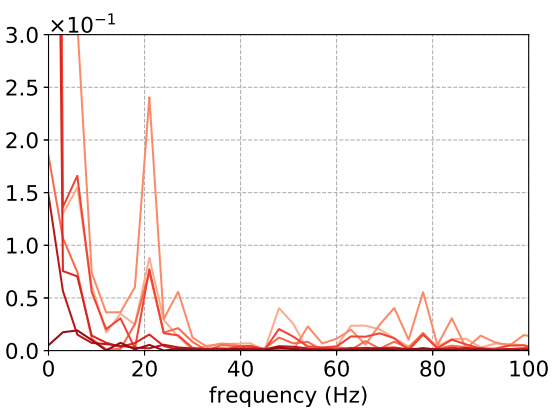

(b) Spectrum of contact

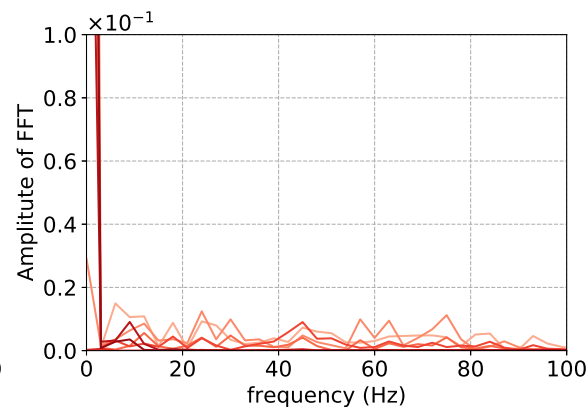

(c) Spectrum of free

Fig. 3. The spectrum of signal segmetns (external toques on 7 joints) respectively with classes of collision 2(a), contact 2(b) and free 2(c) using FFT.

To determine the value of $l$, we inspect the waveform of the pHRI signals presented in Fig. 2. We notice that the average width of a contact is approximately $400 \mathrm{~ms}$, whilst that of a collision is less than $200 \mathrm{~ms}$. This also applies to most of the recorded pHRI signals. Therefore, we set the segment width as $l=300(\mathrm{~ms})$, as a balanced result. Then, we label the two types of segment samples respectively as collision (Fig. 2(a)) and contact (Fig. 2(b)). The segmented parts of the raw signals are marked by colored areas in Fig. 2. During the segmentation, invalid data due to storage damages or unrecognizable pHRI waveforms are eliminated. As a result, we obtain 6718 collisions and 7346 contacts, which is approximate to a balanced radio $1: 1$. We also create signal segments without any pHRI waveform and label them as free. However, the number of free samples should not be equal to the other two classes since the frequency of contact-less cases is usually higher than pHRI. In practice, both collisions and contacts are positive instances which will call the corresponding safety mechanism, whilst frees are negative instances which do not trigger the safety mechanism. Therefore, a reasonable idea would be to keep a balance between the free samples and the summary of all pHRI samples, i.e., a scale ratio 1:1:2 between collision, contact and free. Therefore, we take the free segments respectively in the front, rear and middle of the pHRI segments in the raw signals, and finally obtain 13633 of them.

Until now, a data set containing the pHRI signal segments is constructed. We randomly shuffle all the samples and split them into a training set and a test set with the partition ratio $3: 1$. The training set will be applied to feature engineering and model selection, whilst the test set will be used to evaluate the trained model.

\section{Development of the Signal Classifier}

In this section, we develop the signal classifier component in the CDI scheme. As explained in Sec. II, we do not consider the probabilistic series-models to avoid prior knowledge on the distributional dependence of the raw signals. Additionally, although the end-to-end learning mechanisms, such as the convolutional neural network (CNN) and recurrent neural network (RNN), have drawn much attention and made great achievements in image recognition and model-free planning, they still suffer from the lack of explainability and high dependence on complex manually designed structures. Therefore, in this paper, we develop the signal classifier based on the paradigm of supervised learning, by which we assume that the samples in the data set are independent of each other.

\section{A. Feature Extraction}

To form a feature set that benefits the signal classification, we consider both the properties of pHRI signals and the successful experience in previous work [27], [38]. From Fig. 2, we can conclude that the waveform of collisions (Fig. 2(a)) has 
sharp shapes and fast amplitude changes, while the waveform of contacts (Fig. 2(b)) changes more gently and lasts for a longer time. We also investigate the spectrum of the signal segments in Fig. 2 and shown it in Fig. 3. Compared to the free sample, the collision and contact possess more components in all frequency ranges. Especially, the collision shows a large amplitude in the frequency interval $10-20 \mathrm{~Hz}$, whist the contact in $0-10 \mathrm{~Hz}$. Therefore, distinguishable properties between the two classes are found in both time-domain and frequencydomain. Based on this, we initially extract 18 features in both domains. Most of these features also have achieved success in previous work [38].

1) Features in the time domain: The time domain features are frequently used in the fields like signal processing and pattern recognition [39], [40]. Since each sample is naturally a segmented time series $\mathcal{T}=\left\{\tau_{1}, \tau_{2}, \cdots, \tau_{l}\right\}$, the time-domain features can be represented as the functions of $\mathcal{T}$. Here, we mainly select features that are concerned with the amplitude changes of the signals. First of all, the 1st to 4th order statistical moments, namely the mean value $M_{\tau}$, the variance $V_{\tau}$, the skewness $S_{\tau}$ and the kurtosis $K_{\tau}$ of samples $\mathcal{T}$ are applied to depict the stochastic properties of the signal segment, where $M_{\tau}=\frac{1}{l} \sum_{i=1}^{l} \tau_{i}, K_{\tau}=\frac{1}{l} V_{\tau}^{-2} \sum_{i=1}^{l}\left(\tau_{i}-M_{\tau}\right)^{4}-3, V_{\tau}=$ $\frac{1}{l} \sum_{i=1}^{l}\left(\tau_{i}-M_{\tau}\right)^{2}$ and $S_{\tau}=\frac{1}{l} V_{\tau}^{-\frac{3}{2}} \sum_{i=1}^{l}\left(\tau_{i}-M_{\tau}\right)^{3}$. The median value $\tilde{m}_{\tau}$, the extreme range $R_{\tau}$ and the extreme deviation $D_{\tau}$ of $\mathcal{T}$ are also used as supplementary, where $R_{\tau}=\max _{i}\left|\tau_{i}\right|-\min _{i}\left|\tau_{i}\right|$ and $D_{\tau}=\max _{i}\left|\tau_{i}\right|-M_{\tau}$, for $1 \leqslant i \leqslant l$. Additionally, we propose the energy increasing rate $E_{I}=\frac{1}{2} \lg \left(\sum_{i=[n / 2]}^{n} \tau_{i}^{2} / \sum_{i=1}^{[n / 2]} \tau_{i}^{2}\right)$ to represent the temporal change of signal energy within a segment, where the ratio is made between the squared signal sums within the two halves of the segment. Here, [.] rounds a real number to integer.

2) Features in the frequency domain: The frequency domain features are commonly applied to depict the spectral profiles of signals [11], [41], which are mostly calculated by fast Fourier transformation (FFT). The FFT of a signal sequence $\mathcal{T}$ includes a sampled frequency sequence $\mathcal{F}=\left\{f_{1}, f_{2}, \cdots, f_{m}\right\}$ with the frequency sampling interval $f_{s} / m$, and the corresponding complex spectrum sequence $\mathcal{S}=\left\{S\left(f_{1}\right), S\left(f_{2}\right), \cdots, S\left(f_{m}\right)\right\}$. The length of the spectrum sequence $m$ is usually equal to $l$. The frequency domain features used in this paper are mainly concerned with the amplitudes and phase angles of the signal spectrum in different frequency intervals, such as the mean frequency $\bar{f}$, the fundamental frequency $f^{*}$ and their corresponding spectral amplitudes $|S(\bar{f})|,\left|S\left(f^{*}\right)\right|$ and phase angles $\phi(\bar{f}), \phi\left(f^{*}\right)$, where $|\cdot|: \mathbb{C} \rightarrow \mathbb{R}$ and $\phi(\cdot): \mathbb{C} \rightarrow \mathbb{R}$ respectively denote the amplitudes and phase angles. The mean frequency $\bar{f}$ is defined as $\bar{f}=\sum_{i=1}^{m} f_{i}\left|S\left(f_{i}\right)\right| / \sum_{i=1}^{m}\left|S\left(f_{i}\right)\right|$ and the fundamental frequency $f^{*}$ is the frequency point such that $S\left(f^{*}\right)$ is the spectrum summit. The crest factor $F_{\text {crest }}$, the average signal energy $E_{\mathrm{rms}}$ and the subband energy ratio $E_{r}^{f_{c}}$ are respectively defined as $F_{\text {crest }}=$ $\left|S\left(f^{*}\right)\right| / \sqrt{\frac{1}{m} \sum_{i=1}^{m}\left|S\left(f_{i}\right)\right|^{2}}, \quad E_{\mathrm{rms}}=\sqrt{\frac{1}{m} \sum_{i=1}^{m}\left|S\left(f_{i}\right)\right|^{2}}$, $E_{r}^{f_{c}}=\frac{1}{2} \lg \left(\sum_{f_{i}=f_{c}}^{f_{s}}\left|S\left(f_{i}\right)\right|^{2} / \sum_{f_{i}=0}^{f_{c}}\left|S\left(f_{i}\right)\right|^{2}\right.$ for $f_{c}=$ 10, $20 \mathrm{~Hz}$. These features are also widely used in [38], [39], [42] to depict the energy properties of signals. The crest factor
$F_{\text {crest }}$, also known as peak-to-average ratio, represents the significance of the signal peak. The average energy $E_{\mathrm{rms}}$ denotes the average signal power level, and the sub-band energy ratio $E_{r}^{f_{c}}$ reflect the relative energy proportion on the two sides of the sub-band frequency $f_{c}$. Here, we define two sub-band energy indexes. The corresponding sub-band frequencies $10 \mathrm{~Hz}$ and $20 \mathrm{~Hz}$ are determined according to the distinguishable spectral features of collisions and contacts.

In summary, we extract the following features $\mathcal{X}^{+}=\left\{M_{\tau}\right.$, $V_{\tau}, S_{\tau}, K_{\tau}, \bar{m}_{\tau}, R_{\tau}, D_{\tau}, E_{I}, f^{*},\left|S\left(f^{*}\right)\right|, \phi\left(f^{*}\right), \bar{f},|S(\bar{f})|$, $\left.\phi(\bar{f}), F_{\text {crest }}, E_{\mathrm{rms}}, E_{r}^{10}, E_{r}^{20}\right\}$, where $\mathcal{X}^{+}$denotes a full feature set. For brevity, we order these features and number them from \#1 to \#18. Note that the signals have 7 dimensions corresponding to the 7 robot joints. Therefore, in total 126 features are generated.

\section{B. Feature Selection}

To select the beneficial features for the classification, we use the Spearman correlation analysis and the Relieff algorithm to evaluate the 126 features in $\mathcal{X}^{+}$. The former inspects the dependence between any two features, whilst the latter calculates weights for the features which are recognized as their importance to the classification. Features with high dependence on the others or low importance are eliminated after the analysis. Note that we only use the data in the training set during the entire feature selection procedure.

1) dependence Analysis: High dependence among features brings redundancy to the model training, which potentially causes a poor generalization ability to the classifier. Therefore, we analyze the Spearman correlation between the features to investigate their dependence. The Spearman rank correlation analysis is an efficient tool to describe the general monotonic relationship between two stochastic variables, which applies not only to linear dependence, but also the nonlinear cases. Given the values of two features $X=\left\{X_{1}, X_{2}, \cdots, X_{m}\right\}$ and $Y=\left\{Y_{1}, Y_{2}, \cdots, Y_{m}\right\}$, where $m$ is the size of the data set, the Spearman correlation coefficient $\rho_{X, Y}$ is calculated by $\rho_{X, Y}=$ $\sum_{i=1}^{m}\left(x_{i}-\bar{x}\right)\left(y_{i}-\bar{y}\right) / \sqrt{\sum_{i=1}^{m}\left(x_{i}-\bar{x}\right)^{2} \sum_{i=1}^{m}\left(y_{i}-\bar{y}\right)^{2}}$, where $x=\left\{x_{1}, x_{2}, \cdots, x_{m}\right\}$ and $y=\left\{y_{1}, y_{2}, \cdots, y_{m}\right\}$ are the ranks of features $X$ and $Y$, and - denotes the mean value [43]. Ranging from -1 to 1 , the coefficient $\rho_{X, Y}$ depicts the dependence between $X$ and $Y$, of which the sign denotes whether the relationship of the two variables are proportional (when positive) or inversely proportional (when negative), and the absolute value indicates the extent of dependence. We apply the Spearman correlation analysis to all 126 features in $\mathcal{X}^{+}$. The results are illustrated in Fig. 4 by colored matrices.

Fig. 4(a) displays the dependence between all 126 features which are grouped by the joint numbers. Each square block (surrounded by black dashed lines) on the diagonal represents the self-feature- dependence of each joint, i.e., the dependence between the features of the same joint. Meanwhile, the blocks off the diagonal indicate the dependence among the features of different joints. It is noticed that the the blocks on the diagonal are highly similar to each other, which indicates that the features on all robot joints possess similar self-dependence properties. Therefore, it is reasonable to only investigate the 


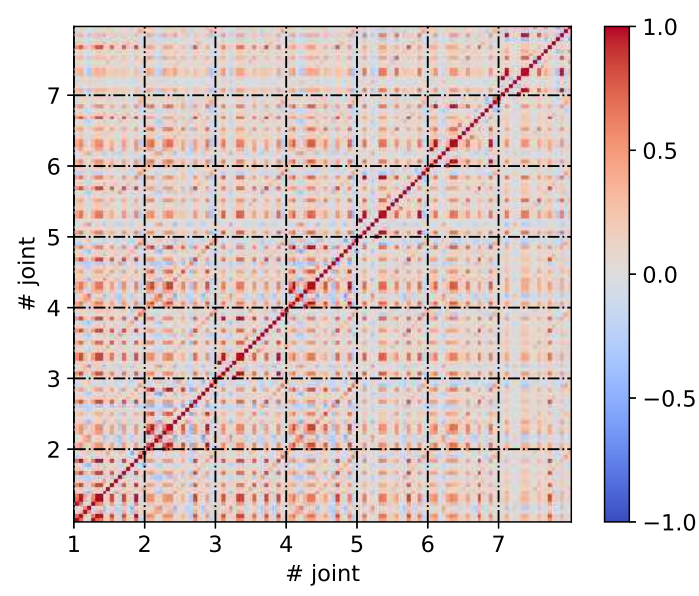

(a) The spearman correlation between the 126 features.

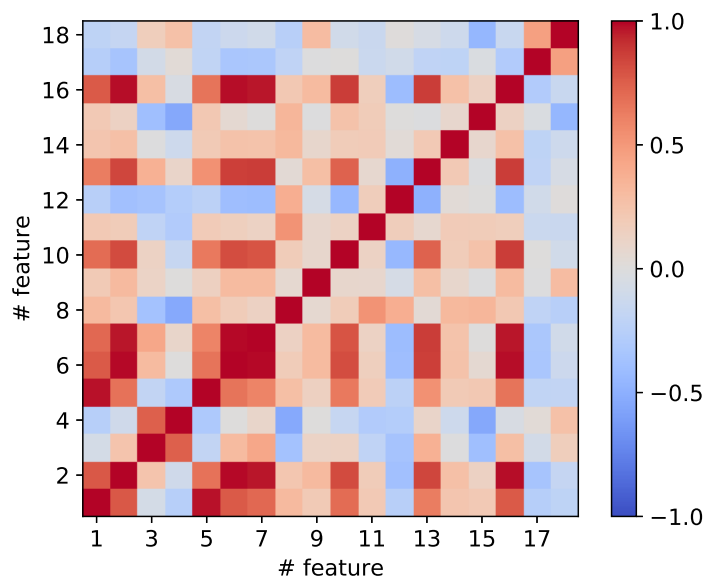

(b) The spearman correlation between the 18 features on joint \# 1 .

Fig. 4. The Spearman correlation analysis of the features. Each micro square block represents the correlation coefficients between the corresponding two features, of which the values are marked by colors. Dark red indicates highly positive dependence and dark blue means the opposite.

analysis result of a single joint and selected or eliminated the features in batches in the unit of joint.

The correlation analysis of all 18 features on joint \#1, which is corresponding the block in the down-left corner in Fig. 4(a), is illustrated in Fig. 4(b). The results reveal that features \# $1\left(M_{\tau}\right)$ and \# $5\left(\bar{m}_{\tau}\right)$ show high dependence, so do features \# $2\left(V_{\tau}\right)$, \# $6\left(R_{\tau}\right)$, \# $7\left(D_{\tau}\right)$ and \# $16\left(E_{\mathrm{rms}}\right)$. Therefore, these features are preferably considered to be eliminated from $\mathcal{X}^{+}$, yet their importance to the classification still needs to be considered.

2) Importance Analysis: After analyzing the dependence of the features, we apply the Relieff algorithm to the 126 features in $\mathcal{X}^{+}$to evaluate their importance. As a popular feature engineering technique used in the previous work [38], it provides a weight for each feature to depict its importance to the classification, which is independent of the classifier models. The predecessor of Relieff, known as Relief, is originally proposed in [44] for binary classification problems.
Its main idea is to recursively and randomly select a sample $x$ from the data set and find out its closest samples with the same and different labels (respectively referred as the near-hit $z^{+}$and the near-miss $z^{-}$). For the $i$-th feature of sample $x$, $x_{i}$, its weight $w_{i}$ is iteratively updated by the following law,

$$
w_{i}=w_{i}-\left(x_{i}-z_{i}^{+}\right)^{2}+\left(x_{i}-z_{i}^{+}\right)^{2},
$$

where $z_{i}^{+}$and $z_{i}^{-}$are the $i$-th features of $z^{+}$and $z^{-}$. The weight $w_{i}$ depicts the reward (if $w_{i}$ is positive) or punishment (if $w_{i}$ is negative) of the $i$-th feature to the classification, and a larger $w_{i}$ indicates higher importance. Relieff is adapted from Relief as the extension to multi-class problems [45].

On the training set, we calculate the weights, or importance of all 126 features using Relieff and illustrate the results in Fig. 5, grouping them respectively by the feature number (see Fig. 5(a)) and joint number (see Fig. 5(b)). The results indicate that the feature importance varies among both features and joints. In Fig. 5(a), the feature \#2 $\left(D_{\tau}\right)$ shows the lowest importance (0.49), followed by \#4 $\left(K_{\tau}\right)$, \# $5\left(\bar{m}_{\tau}\right)$, \#9 $\left(f^{*}\right)$, and \#13(S|(f) $) \mid)$. In Fig. 5(b), the feature weights on joint \# 1, \# 2 and \# 4 are higher than the other joints.

Since the classifier is supposed to function in a online scheme, we are quite concerned with the computational load of the features. Therefore, we tend to form a small feature set, while maintaining a decent classification accuracy. Considering both high dependence and low importance, we eliminate features \#2 $\left(D_{\tau}\right)$, \# $4\left(K_{\tau}\right)$ and \# $5\left(\bar{m}_{\tau}\right)$. Features \# $6\left(R_{\tau}\right)$ and $\# 16\left(E_{\mathrm{rms}}\right)$ are also eliminated due to their strong relationship with \# $7\left(D_{\tau}\right)$, as well as \# $18\left(E_{r}^{20}\right)$ due to its redundancy to $\# 17\left(E_{r}^{10}\right)$. Additionally, we remove the features of the joints \#3, \# 5, \#6 and \#7 due to their low importance. Therefore, we determine the feature set for the development of the signal classifier as $\mathcal{X}^{*}=\left\{M_{\tau}, S_{\tau}, D_{\tau}, E_{I}, f^{*},\left|S\left(f^{*}\right)\right|, \phi\left(f^{*}\right), \bar{f}\right.$, $\left.|S(\bar{f})|, \phi(\bar{f}), F_{\text {crest }}, E_{r}^{10}\right\}_{1,2,4}$ which contains 36 features, where the subscript denotes the joints. To evaluate $\mathcal{X}^{*}$, we also define a minimum set $\mathcal{X}^{-}=\left\{M_{\tau}, S_{\tau}, D_{\tau}, E_{I}, f^{*},\left|S\left(f^{*}\right)\right|\right.$, $\left.\phi\left(f^{*}\right), \bar{f},|S(\bar{f})|, \phi(\bar{f}), F_{\text {crest }}, E_{r}^{10}\right\}_{1}$, which only includes 12 feature on joint \#2. We will evaluate the three feature sets $\mathcal{X}^{+}$, $\mathcal{X}^{*}$ and $\mathcal{X}^{-}$and select the best when determining the classifier models. Note that such development procedure is justified since the entire feature selection process is independent from the classification models.

\section{Model Validation}

After determining the feature sets $\mathcal{X}^{+}, \mathcal{X}^{*}$ and $\mathcal{X}^{-}$, we are ready to select the model for the signal classifier. Since a large number of models are used in the literature for the classification of time series, enumerating all of them for the best one is a tedious and unnecessary process. Instead, we assign several popular models with representative properties as candidates, and validate these candidate models using the training set, before selecting the best one. In this paper, we determine the candidate models based on three factors, namely the pHRI signal properties, engineering experience in the previous work and the values of models in practice. Thus, four classifier models, namely a linear discriminant analysis (LDA) model, a k-nearest neighbors (kNN) model, an SVM 


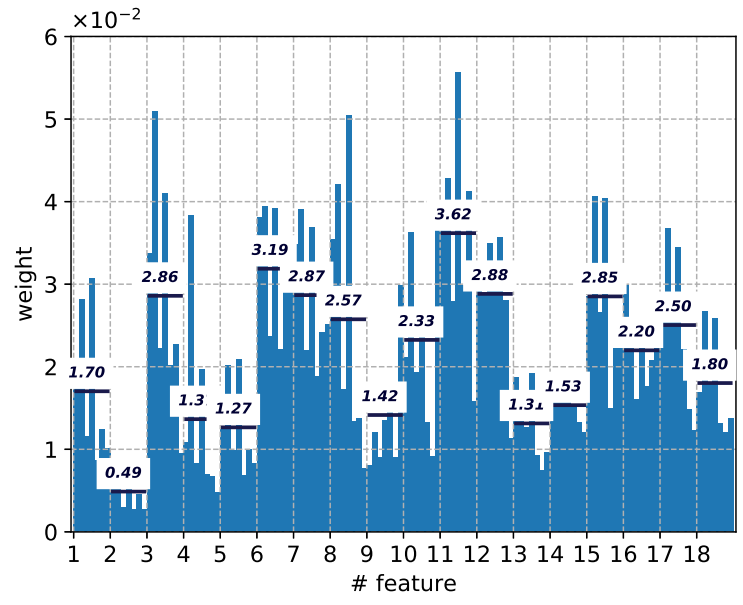

(a) Feature weights grouped by feature numbers. The averaged weight over all 7 joints is marked for each feature.

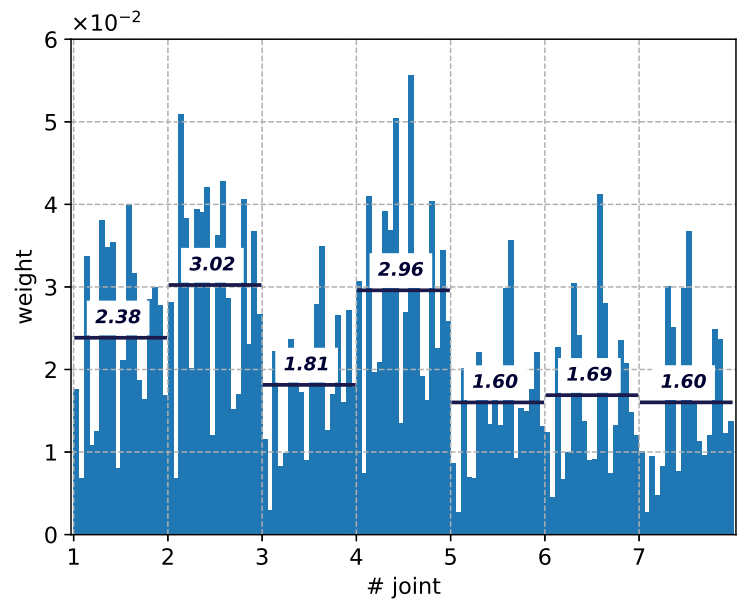

(b) Feature weights grouped by joint numbers. The averaged weight over all features is marked for each joint.

Fig. 5. The weights of the features obtained by Relieff algorithm.

model, and a feedforward neural network (FNN) model are chosen as candidate models, due to their simple structures and successful applications in the previous work [38]. These four models are featured with various properties and essentially cover most representative classifier structures. Specifically, the LDA model is well known for its high efficiency and stable performance, and is able to achieve high prediction accuracy for the linearly classifiable data sets. The kNN model is supported by well-developed theoretical foundations and is easy to be extended to a larger scale database in future work. The SVM model shows the excellent ability of generalization. The $\mathrm{NN}$ model, as a powerful and comprehensive approximator, has strong adaptability and flexibility for various data sets. In this paper, we only consider the NN model with one hidden layer. Note that simple structures are always preferred in practice since the reliability of the system tends to decrease when the system becomes more complicated. To evaluate the four classifier models on the training set with the feature sets $\mathcal{X}^{+}, \mathcal{X}^{*}$ and $\mathcal{X}^{-}$, we use a ten-fold cross-validation method and calculate the mean values of the ten validation scores which are briefly referred to as score. We also apply a grid search method to seek for the best hyper-parameters of the models. The models and the corresponding grid values of their hyper-parameters are shown in Tab. I.

\section{TABLE I}

HYPER-PARAMETERS FOR GRID-SEARCHING VALIDATION

\begin{tabular}{|c|c|c|}
\hline Models & Hyper-params & Grid values \\
\hline LDA & solver & 'svd', 'lsqr', 'eigen' \\
\hline & $k$ value & $2,3, \cdots, 80$ \\
\hline $\mathrm{kNN}$ & distance metrics & $\begin{array}{l}\text { 'canberra', 'chebyshev', 'euclidean', } \\
\text { 'manhattan', 'minkowski' }\end{array}$ \\
\hline \multirow{2}{*}{ SVM } & $C$ value & $10^{-3}, 10^{-2.8}, \cdots, 10^{3}$ \\
\hline & kernel function & 'linear', 'rbf', 'sigmoid' \\
\hline FNN & $\begin{array}{l}\text { hidden neurons } \\
\text { activation function }\end{array}$ & $\begin{array}{l}2,4, \cdots, 100 \\
\text { 'identity', 'logistic', 'tanh', 'relu' }\end{array}$ \\
\hline
\end{tabular}

The four models and the corresponding hyper-parameter values achieving the best scores in the validation are listed in Tab. II, where the hyper-parameter values are in the same order as the hyper-parameters in Tab. I. The validation scores of the models trained with various feature sets are presented in the score columns, $\mathcal{X}^{+}, \mathcal{X}^{*}$ and $\mathrm{X}^{-}$, respectively, which show that all four models achieve satisfactory scores, with the FNN model showing the highest (96.6\%). Thus, we select FNN with 48 hidden-layer neurons, with 'logistic' activation function as the model for the signal classifier of the CDI scheme. In the meantime, the results also reveal that the scores using $\mathcal{X}^{*}$ are close to that using the full feature set $\mathcal{X}^{+}$for all models, despite of slight decreases. On the contrary, the scores of the minimum feature set $\mathcal{X}^{-}$drastically drop compared to $\mathcal{X}^{+}$ $\mathcal{X}^{*}$. Therefore, we determine $\mathcal{X}^{*}$ as the feature set to train the FNN model, since it achieves similar scores to the full feature set $\mathcal{X}^{+}$with a greatly reduced number of features.

TABLE II

THE MODEL CONFIGURATIONS WITH BEST VALIDATION SCORES

\begin{tabular}{ccccc}
\hline Models & Hyper-parameters & $\operatorname{score}\left(\mathcal{X}^{+}\right)$ & $\operatorname{score}\left(\mathcal{X}^{*}\right)$ & $\operatorname{score}\left(\mathcal{X}^{-}\right)$ \\
\hline LDA & 'svd' & $94.8 \%$ & $92.4 \%$ & $82.2 \%$ \\
kNN & 5, 'canberra' & $97.5 \%$ & $96.3 \%$ & $88.0 \%$ \\
SVM & 63.1, 'linear' & $97.3 \%$ & $96.0 \%$ & $86.4 \%$ \\
FNN & 48, 'logistic' & $96.8 \%$ & $96.6 \%$ & $89.7 \%$ \\
\hline
\end{tabular}

\section{Model Test}

After determining the model for the signal classifier (FNN, 48 hidden-layer neurons, 'logistic' activation function), we train the model using all samples in the training set with the feature set $\mathcal{X}^{*}$ and evaluate its prediction accuracy using the test set. The tested accuracy score, $96.5 \%$, is generally comparable with the score $96.6 \%$ in the model validation in Sec. IV-C, which indicates that the signal classifier possesses a satisfactory ability of generalization. The confusion matrix 
of the test is presented in Fig. 6, where the prediction accuracy for collisions, contacts and free respectively achieves as high as $95.6 \%, 93.7 \%$ and $98.4 \%$, showing a decent performance of the classifier. Especially, the higher accuracy rate of collisions than contacts reveals that the classifier is more sensitive to accidental collisions, which is reasonable since collisions are more dangerous to humans than contacts. The high accuracy score for free means that the classifier hardly produces falsepositive results.

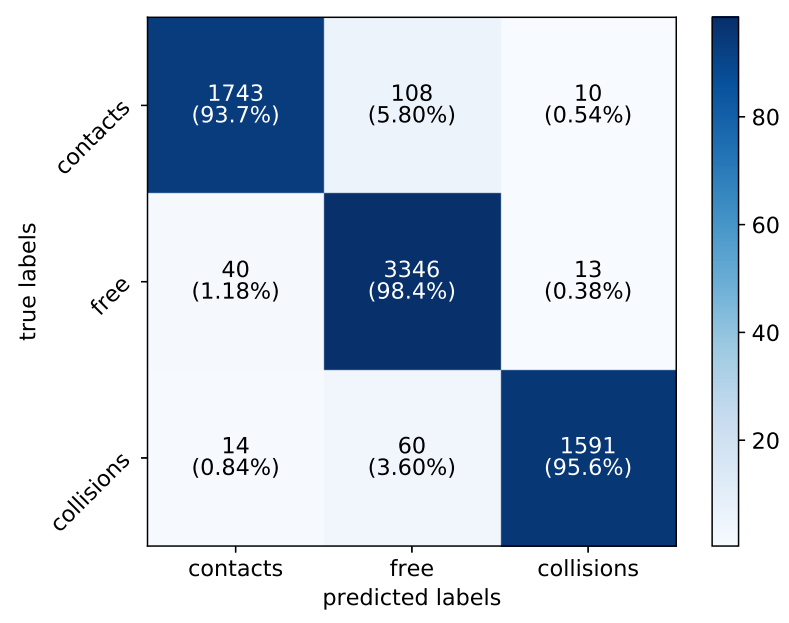

Fig. 6. The confusion matrix in the test of the well trained FNN classifier.

\section{Design of the Online Diagnosor}

Although the signal classifier shows high accuracy for samples with complete pHRI waveform, the same performance is not likely to be ensured in the early stage of a pHRI in online applications, since the segmented waveform is incomplete. As discussed in Sec. I, the main reason is the distribution inconsistency between the training set and the segmented online signals, such that a single prediction result of the classifier does not guarantee a reliable CDI before the pHRI vanishes. To resolve this issue, we design an additional online diagnosor for the CDI scheme to produce online decisions based on a series of prediction results, which is proven to be more reliable than using a single result.

\section{A. Prediction Analysis of Incomplete pHRI Waveform}

As the first step of designing the online diagnosor, we investigate the prediction accuracy of the signal classifier for signal samples with incomplete pHRI waveform. We create several test sets from the raw signals with various $b$ values which represent the segmented online signals with different proportion of pHRI waveform, and use them to evaluate the prediction accuracy of the signal classifier. Note that all the test sets are of the same size as the one in Sec. IV-D. The accuracy scores with different $b$ values are illustrated in Fig. 7.

As a general tendency, the prediction accuracy increases when the segment bias $b$ becomes larger. An intuitive explanation is that more useful information on the pHRI waveform naturally leads to higher accuracy scores. Within the interval $b \leqslant 50$, the prediction accuracy is lower than $77.3 \%$, which indicates that a prediction result within $50 \mathrm{~ms}$ after the pHRI is very likely to be incorrect. In contrast, when $b>200$, the accuracy is higher than $93.0 \%$, which reveals that an accurate result is only possible after $200 \mathrm{~ms}$. Apparently, a single prediction result of the signal classifier does not offer a reliable CDI decision in the early stage of pHRI.

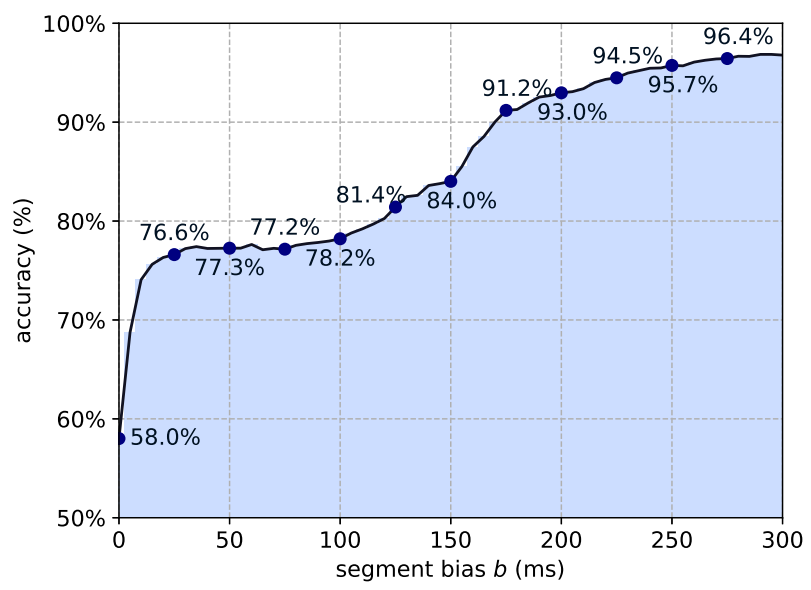

Fig. 7. The accuracy scores of the signal classifier when predicting samples with various $b$ values.

\section{B. Diagnosis Using Confidence Indexes}

Our solution to this problem is to extend the decision horizon, such that the CDI result $C^{*}$ is produced based on a series of successive prediction results. Moreover, a quantified metric is need to depict the reliability of the CDI result. Let us assume that the pHRI occurs at time $t_{0}$. At a certain time instant $t_{1}=t_{0}+b$, a segmented torque signal $\mathcal{T}_{1}$ is given to the signal classifier which then reports a prediction $r_{1}$. The value of the prediction result $r_{1}$ can be $-1,0$ or 1 which corresponds to collision, contact and free. Here, $r_{1}$ is not directly used to make the decision $C^{*}$. Instead, we postpone $s$ sampling intervals until we obtain $s$ signal segments $\mathcal{T}_{1}, \mathcal{T}_{2}, \cdots, \mathcal{T}_{s}$ and a series of prediction results $\mathcal{R}=\left\{r_{1}, r_{2}, \cdots, r_{s}\right\}$. Here, we refer to $\mathcal{R}$ as an observation series with the window size $s$. According to the Bayesian decision theory, the posterior probability of the class $C=\{-1,0,1\}$ based on the observation series $\mathcal{R}$ with parameter $b$ reads

$$
p(C \mid \mathcal{R}, b)=\frac{p(C \mid b) p(\mathcal{R} \mid C, b)}{p(\mathcal{R} \mid b)},
$$

where $p(C \mid b)$ is the prior knowledge of proportions of the data set, $p(\mathcal{R} \mid C, b)$ is the likelihood of class $C$ and $p(\mathcal{R} \mid b)$ is the evidence that adjusts the weight of the posterior probabilities, where $p(\mathcal{R} \mid b)=\sum_{C} p(C \mid b) p(\mathcal{R} \mid C, b)$. As mentioned in the beginning of Sec. IV, the signal segments $\mathcal{T}_{1}, \mathcal{T}_{2}, \cdots, \mathcal{T}_{s}$ are independent from each other, so are the prediction results $r_{1}, r_{2}, \cdots, r_{s}$. Therefore, the likelihood $p(\mathcal{R} \mid C, b)$ can be calculated by the following product

$$
p(\mathcal{R} \mid C, b)=\prod_{j=1}^{s} p\left(r_{j} \mid C, b_{j}\right),
$$


where for each $r_{j}, j=1,2, \cdots, s, p\left(r_{j} \mid C, b_{j}\right)$ represents the likelihood of a single prediction result $r_{j}$ with given pHRI class $C$. The parameter $b_{j}$ is the bias of segment $\mathcal{T}_{j}$, reflecting a hidden condition that $r_{j}$ is the $j$-th element of $\mathcal{R}$. The likelihood $p\left(r_{j} \mid C, b_{j}\right)$ depicts the probability that the classifier predicts a sample labeled $C$ as $r_{j}$, which depends on the capability of the classifier.

Here, the posterior probability $p(C \mid \mathcal{R}, b)$ serves as a confidence index for decision $C$ given observation series $\mathcal{R}$. It indicates the belief on the classifier to produce an accurate diagnosis result within $b \mathrm{~ms}$ after the occurrence of the pHRI. A larger value of $p(C \mid \mathcal{R}, b)$ corresponds to a more trustful decision $C$. Therefore, the decision law for the online diagnosor can be designed as

$$
C^{*}=i, \text { if } p\left(C^{[i]} \mid \mathcal{R}, b\right)=\max _{C} p(C \mid \mathcal{R}, b), i=-1,0,1,
$$

where $C^{*}$ is the diagnosis result $\left(C^{*}=-1\right.$ for contacts, $C^{*}=$ 1 for collisions and $C^{*}=0$ for free) and $C^{[i]}$ represents the event $C=i$ for brevity. The confidence index for decision $C^{*}$ is $\mathcal{I}_{\mathcal{R}}^{C^{*}}=p\left(C^{*} \mid \mathcal{R}, b\right)$.

To calculate the posterior probabilities, the likelihoods $p\left(r_{j} \mid C, b_{j}\right)$ for all $r_{j}=\{-1,0,1\}$ and $C=\{-1,0,1\}$ can be approximated by experimental methods similar to that in Sec. V-A. The difference is that the calculation of the likelihoods requires the values in the confusion matrices, rather than the overall accuracy scores. Such approximation is justified by the large number principle. The observation window size $s$ can be determined accordingly. A wider window leads to a higher resolution of the confidence index and provides more flexibility to the collision handling pipeline, which on the other hand, involves more computational load. Note that the value $b$, as a parameter to be assigned, represents a conservative estimation, rather than the true diagnosis delay. A reliable CDI diagnosis is guaranteed in the early stage of a pHRI, as long as a high confidence index is obtained for even small values of $b$. Therefore, in the practical design of the CDI scheme, $b$ can be heuristically determined as a reference reaction time. By designing the decision law (3) with the confidence index $\mathcal{I}$, the collision handling pipeline can be implemented in a more flexible manner.

\section{A Fast Online Diagnosis Algorithm}

The decision law in (3) requires the calculation of the posterior probabilities for every observation series $\mathcal{R}$, which is not suitable for online applications due to the large computational load. Here, we propose a fast online diagnosis algorithm (as Algorithm 1) that only involves simple comparison logic but ensures high reliability. In the algorithm, the observation window size is set as $s=5$. The main routine of the algorithm is to compare the observation $\mathcal{R}$ with two reference events $\mathcal{R}_{l}^{4}$ and $\mathcal{R}_{t}^{2}$, where $\mathcal{R}_{l}^{4}$ means that at least 4 collisions are observed in $\mathcal{R}$, and $\mathcal{R}_{t}^{2}$ represents at least 2 contacts are witnessed. To evaluate the reliability of Algorithm 1, we calculate its posterior probabilities, $p\left(C^{[1]} \mid \mathcal{R}_{l}^{4}, b\right), p\left(C^{[1]} \mid \mathcal{R}_{t}^{2}, b\right)$ and $p\left(C^{[1]} \mid \mathcal{R}_{f}, b\right)$ with $b=50$, where $\mathcal{R}_{f}$ denotes $\overline{\mathcal{R}_{l}^{4} \vee \mathcal{R}_{t}^{2}}$, i.e., neither a collision nor a contact occurs. Here, $\vee$ denotes the union of two events and $\overline{(\cdot)}$ represents the compliment of a event. Therefore, the posterior $p\left(C^{[1]} \mid \mathcal{R}_{l}^{4}, b\right)$ represents the confidence index of Algorithm 1 when making a decision $C=1$ given $\mathcal{R} \in \mathcal{R}_{l}^{2}, p\left(C^{[1]} \mid \mathcal{R}_{l}^{4}, b\right)$ is for decision $C=-1$ with $\mathcal{R}_{t}^{2}$, and the else is depicted by $p\left(C^{[0]} \mid \mathcal{R}^{f}, b\right)$ which describes the reliability of the algorithm in free cases.

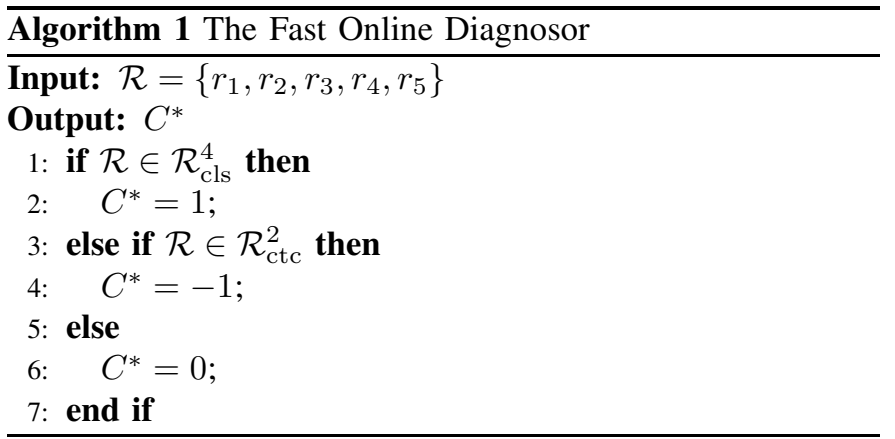

To calculate the posteriors, we firstly use (2) to calculate the following likelihoods,

$$
\begin{gathered}
p\left(\mathcal{R}_{l}^{4} \mid C^{i}, b_{j}^{[50]}\right)=\sum_{\mathcal{R} \in \mathcal{R}_{l}^{4}} \prod_{j=1}^{s} p\left(r_{j} \mid C^{i}, b_{j}^{[50+j]}\right), i=[1], \overline{[1]} \\
p\left(\mathcal{R}_{t}^{2} \mid C^{i}, b_{j}^{[50]}\right)=\sum_{\mathcal{R} \in \mathcal{R}_{t}^{2}} \prod_{j=1}^{s} p\left(r_{j} \mid C^{i}, b_{j}^{[50+j]}\right), i=[-1], \overline{[-1]}, \\
p\left(\mathcal{R}_{f} \mid C^{i}, b_{j}^{[50]}\right)=\sum_{\mathcal{R} \in \mathcal{R}_{f}} \prod_{j=1}^{s} p\left(r_{j} \mid C^{i}, b_{j}^{[50+j]}\right), i=[0], \overline{[0]},
\end{gathered}
$$

where $b^{[50]}$ means $b=50$ and $C^{\overline{[1]}}$ denotes the event that $C \neq 1$, i.e., $C^{\overline{[1]}}=C^{[-1]} \vee C^{[0]}$, while similar is $C^{\overline{[-1]}}$. Such a compact form is for the purpose of brevity. We assume that $p\left(r_{j} \mid C^{[1]}, b_{j}^{[50+j]}\right) \approx p\left(r_{j} \mid C^{[1]}, b_{j}^{[50]}\right)$, for all $j=1,2, \cdots, 5$, considering a high sampling rate of the system. Therefore, the likelihoods are calculated by follows,

$$
\begin{gathered}
p\left(\mathcal{R}_{l}^{4} \mid C^{i}\right)=p\left(r^{[1]} \mid C^{i}\right)^{5}+5 p\left(r^{[1]} \mid C^{i}\right)^{4} p\left(r^{\overline{[1]}} \mid C^{i}\right), \\
p\left(\mathcal{R}_{t}^{2} \mid C^{i}\right)=1-p\left(r^{[-1]} \mid C^{i}\right)^{5}-5 p\left(r^{[-1]} \mid C^{i}\right)^{4} p\left(r^{[-1]} \mid C^{i}\right), \\
p\left(\mathcal{R}_{f} \mid C^{i}\right)=p\left(r^{[0]} \mid C^{i}\right)^{5}+5 p\left(r^{[0]} \mid C^{i}\right)^{4} p\left(r^{[1]} \mid C^{i}\right) \\
+10 p\left(r^{[0]} \mid C^{i}\right)^{3} p\left(r^{[1]} \mid C^{i}\right)^{2}+10 p\left(r^{[0]} \mid C^{i}\right)^{2} p\left(r^{[1]} \mid C^{i}\right)^{3} \\
+5 p\left(r^{[-1]} \mid C^{i}\right) \times\left(p\left(r^{[0]} \mid C^{i}\right)^{4}+4 p\left(r^{[0]} \mid C^{i}\right)^{3} p\left(r^{[1]} \mid C^{i}\right)\right. \\
\left.+6 p\left(r^{[0]} \mid C^{i}\right)^{2} p\left(r^{[1]} \mid C^{i}\right)^{2}+4 p\left(r^{[0]} \mid C^{i}\right) p\left(r^{[1]} \mid C^{i}\right)^{3}\right),
\end{gathered}
$$

respectively for $i=[1], \overline{[1]}, i=[-1], \overline{[-1]}$ and $i=[0], \overline{[0]}$, where $b^{[50]}$ and the subscript $j$ for $r_{j}$ are omitted for brevity. Similar to above, we use $r^{[1]}$ and $r^{\frac{j}{[1]}}$ to represent $r=1$ and $r \neq 1$. To obtain the values of the single-prediction likelihoods on the right sides of (4), we evaluate the signal classifier using the test set with $b=50(\mathrm{~ms})$ and calculate the confusion matrix which is decomposed to three matrices in Tab. III, respectively for the calculation of (4a), (4b) and (4c). The rows of Tab. III $C^{[i]}, i=-1,0,1$ represent the ground truth, whilst the columns $r^{[i]}$ denote the predicted classes. In each block, both the number of samples and the accurate scores are presented, which explicitly corresponds to the values of the 
TABLE III

THE CONFUSION MATRICES OF SIGNAL CLASSIFIER WITH TEST SET $b=50 \mathrm{MS}$

\begin{tabular}{c|cc||c|cc||c|ccc}
\hline$p(r \mid C)$ & $r^{[1]}$ & $r^{\overline{[1]}}$ & $p(r \mid C)$ & $r^{[-1]}$ & $r^{\overline{[-1]}}$ & $p(r \mid C)$ & $r^{[0]}$ & $r^{[-1]}$ & $r^{[1]}$ \\
\hline$C^{[1]}$ & $1207(72.5 \%)$ & $458(27.5 \%)$ & $C^{[-1]}$ & $593(31.9 \%)$ & $1268(68.1 \%)$ & $C^{[0]}$ & $3376(99.4 \%)$ & $20(5.89 \%)$ & $3(0.09 \%)$ \\
$C^{\overline{[1]}}$ & $13(0.25 \%)$ & $5247(99.7 \%)$ & $C^{\overline{[-1]}}$ & $419(8.27 \%)$ & $4645(91.8 \%)$ & $C^{[0]}$ & $1317(37.4 \%)$ & $992(28.2 \%)$ & $1217(34.6 \%)$ \\
\hline
\end{tabular}

likelihoods $p(r \mid C)$. For example, the value of $p\left(r^{[1]} \mid C^{[1]}\right)$ is approximated as 0.73 corresponding to the value in $C^{[1]}, r^{[1]}$.

Checking the values in Tab. III and using (4), we calculate the values of the likelihoods as

$$
\begin{aligned}
p\left(\mathcal{R}_{l}^{4} \mid C^{[1]}\right) & =0.87, p\left(\mathcal{R}_{l}^{4} \mid C^{\overline{[1]}}\right)=9.80 \times 10^{-5} \\
p\left(\mathcal{R}_{t}^{2} \mid C^{[-1]}\right) & =0.51, p\left(\mathcal{R}_{t}^{2} \mid C^{\overline{[-1]}}\right)=6.74 \times 10^{-2}, \\
p\left(\mathcal{R}_{f} \mid C^{[0]}\right) & =0.99, p\left(\mathcal{R}_{f} \mid C^{[0]}\right)=0.52
\end{aligned}
$$

Here, we set the prior probabilities as $p\left(C^{[0]}\right)=0.5$, $p\left(C^{[1]}\right)=p\left(C^{[-1]}\right)=0.25$, according to the proportion of samples in the data set. Therefore, according to the Bayes law in (2), we calculate the confidence indexes for Algorithm 1 as follows,

$$
\begin{aligned}
p\left(C^{[1]} \mid \mathcal{R}_{l}^{4}\right) & =\frac{p\left(C^{[1]}\right) p\left(\mathcal{R}_{l}^{4} \mid C^{[1]}\right)}{\sum_{i=[1]}^{[1]} p\left(C^{i}\right) p\left(\mathcal{R}_{l}^{4} \mid C^{i}\right)}, \\
p\left(C^{[-1]} \mid \mathcal{R}_{t}^{2}\right) & =\frac{p\left(C^{[-1]}\right) p\left(\mathcal{R}_{t}^{2} \mid C^{[-1]}\right)}{\sum_{i=[-1]}^{[-1]} p\left(C^{i}\right) p\left(\mathcal{R}_{t}^{2} \mid C^{i}\right)}, \\
p\left(C^{[0]} \mid \mathcal{R}_{f}\right) & =\frac{p\left(C^{[0]}\right) p\left(\mathcal{R}_{f} \mid C^{[0]}\right)}{\sum_{i=[0]}^{[0]} p\left(C^{i}\right) p\left(\mathcal{R}_{f} \mid C^{i}\right)}
\end{aligned}
$$

which leads to $p\left(C^{[1]} \mid \mathcal{R}_{l}^{4}\right)=0.99, p\left(C^{[-1]} \mid \mathcal{R}_{t}^{2}\right)=0.72$ and $p\left(C^{[0]} \mid \mathcal{R}_{f}\right)=0.66$.

The results of the posterior probabilities indicate that Algorithm 1 produces high confidence on its diagnosis for collisions $\left(\mathcal{I}_{\mathcal{R}}^{1}=0.99\right)$ and contacts $\left(\mathcal{I}_{\mathcal{R}}^{-1}=0.72\right)$ with given observation series $\mathcal{R}$. A larger value of $p\left(C^{[1]} \mid \mathcal{R}_{l}^{4}\right)$ than $p\left(C^{[-1]} \mid \mathcal{R}_{t}^{2}\right)$ means that the diagnosor is more sensitive to collisions than contacts. The confidence index $\mathcal{I}_{\mathcal{R}}^{0}=0.66$ for free reveals that the diagnosor is also trustful for avoiding false detection. Due to the fact that $p\left(C^{[1]} \mid \mathcal{R}_{l}^{4}\right)=\max _{C} p\left(C \mid \mathcal{R}_{l}^{4}\right), p\left(C^{[-1]} \mid \mathcal{R}_{t}^{2}\right)=$ $\max _{C} p\left(C \mid \mathcal{R}_{t}^{2}\right)$ and $p\left(C^{[0]} \mid \mathcal{R}_{f}\right)=\max _{C} p\left(C \mid \mathcal{R}_{f}\right)$, Algorithm 1 is consistent with the decision law in (3). Therefore, the confidence index $\mathcal{I}$ can be explicitly obtained without calculation, which leads to a faster and easier implementation for online applications.

\section{Comparison with the Single Prediction}

To justify the advantage of the online diagnosor using observation series instead of a single prediction result, we also calculate the confidence indexes for the latter for comparison. The confidence index for class $C^{i}$, with a single observation $r^{i}$, is calculated by

$$
p\left(C^{i} \mid r^{i}\right)=\frac{p\left(C^{i}\right) p\left(r^{i} \mid C^{i}\right)}{\sum_{k=i}^{\bar{i}} p\left(C^{k}\right) p\left(r^{k} \mid C^{k}\right)}, i=[-1],[0],[1] .
$$

Checking the likelihoods in Tab. III, we obtain $p\left(C^{[-1]} \mid r^{[-1]}\right)=0.57, p\left(C^{[0]} \mid r^{[0]}\right)=0.75, p\left(C^{[1]} \mid r^{[1]}\right)=0.99$. The results show that such a scheme ensures a low reliability on identifying contacts (0.57), i.e., the diagnosor is sensitive to collisions but quite dull to contacts, which shows a inferior performance than Algorithm 1.

\section{EXPERIMENTAL VALIDATION}

In this section, we evaluate the performance of the proposed CDI scheme in terms of both the identification accuracy and the responsiveness by applying it to an online validation on the recorded raw signals. These signals contain 242 collisions and 225 contacts and have never been used during the development of the signal classifier. The torque signal is sequentially sampled at the rate of $1 \mathrm{kHz}$ and recursively segmented as new samples for the signal classifier. The online diagnosor is equipped with the fast online diagnosor in Algorithm 1 to provide online diagnosis. Note that the evaluation method and metrics in this experiment are different from the model test in Sec. IV-D. For the classifier development, the accuracy scores are calculated based on the number of signal segments containing the pHRI waveform, while in a human-robot collaboration, a CDI scheme focuses more on the number of correctly identified collisions or contacts. The main difference is that the pHRI, in an online application, usually produces several segments with various $b$ values, corresponding to the signals segmented in different sampling instants. Therefore, for the experimental validation, we must clarify that we evaluate the performance of the CDI scheme with respect to the entire pHRI, rather than its segments. We recognize that the pHRI is accurately identified, if the scheme proposes a correct diagnose before it vanishes.

During the experiment, 240 out of 242 collisions and all 225 interactions are correctly identified, with 2 collisions misclassified as 1 contact and 1 free, achieving an overall accuracy $99.6 \%$. Some identification instances are shown in Fig. 8, which illustrates the accuracy and responsiveness of the scheme. It is noticed that most of the diagnosis results are correctly produced in the early stages of the pHRI, which confirms the high accuracy of the online CDI scheme. The fast response of the scheme is also clearly reflected in Fig. 9 with both instances of a collision and a contact. The two pHRI are all correctly identified within $20 \mathrm{~ms}$ after their occurrence, which confirms the responsiveness of the scheme.

To demonstrate the applicability of the proposed online CDI scheme in a practical task, we implement it on the Kuka LWR4+ robot platform (as shown in Fig. 10) together with a collision reaction routine. The task is intended to surveil 


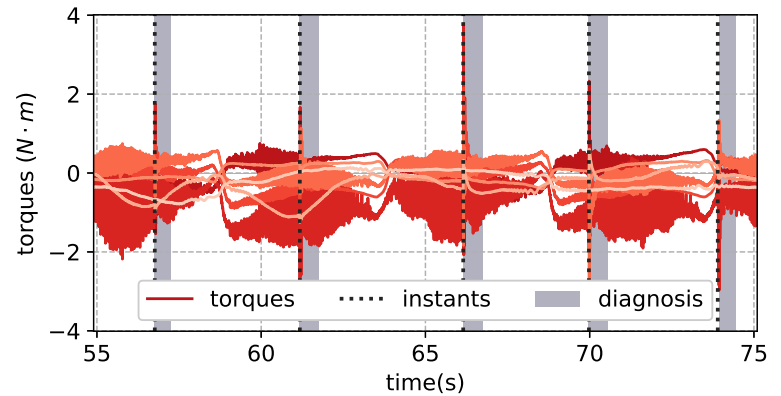

(a) The CDI diagnosis of collisions. The red solid lines are the raw signals, the black dot lines are the occurrence instants of the collisions and the grey regions represent the time intants when collisions are detected and identified.

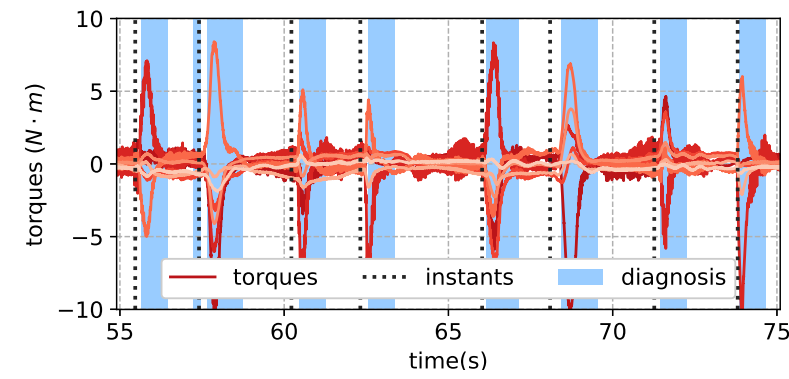

(b) The CDI diagnosis of contacts. The red solid lines are the raw signals, the black dot lines are the occurrence instants of the contacts, and the blue regions represent the time intants when contacts are detected and identified.

Fig. 8. The experiment results of the online CDI scheme respectively for collisions and contacts.

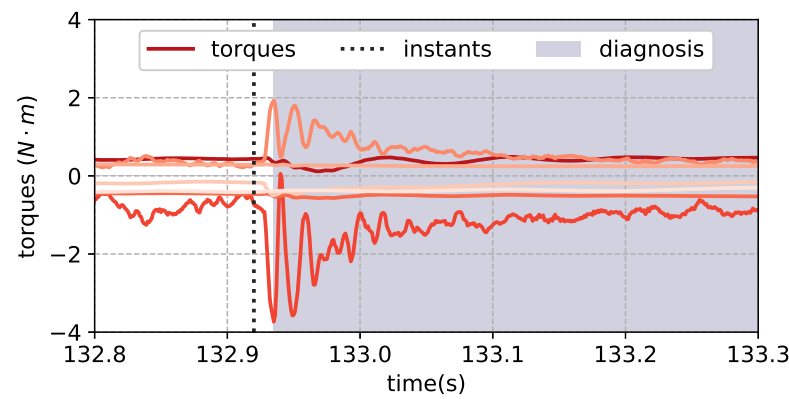

(a) The CDI diagnosis of a collision. The red solid lines are the raw signals, the black dot lines are the occurrence instants of the collisions and the grey regions represent the time intants when collisions are detected and identified.

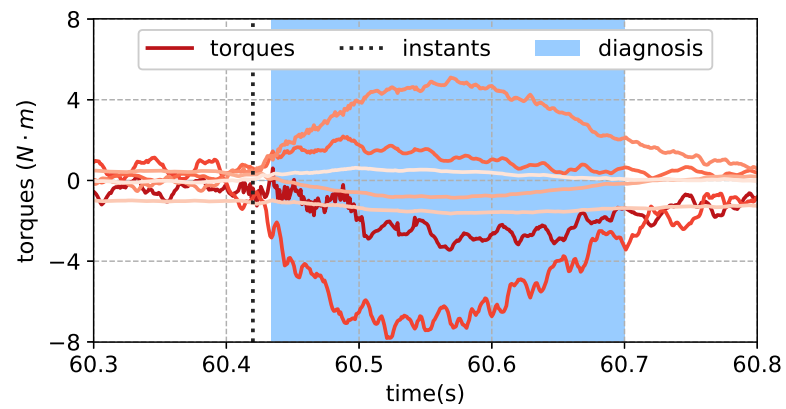

(b) The CDI diagnosis of a contact. The red solid lines are the raw signals, the black dot lines are the occurrence instants of the contacts, and the blue regions represent the time intants when contacts are detected and identified.

Fig. 9. The diagnosis results of a collision and a contact. the external torques on the robot joints, detect any pHRI and activate the corresponding reaction strategies. During a robot task routine, if a collision is detected (as Fig. 10(a)), an emergency stop is triggered and the robot holds on the current position until the safety alarm is deactivated (as Fig. 10(b)). If a contact is detected (as Fig. 10(c)), the robot enables its gravity compensation mode such that the human can conduct active guidance on the robot (as Fig. 10(d)).

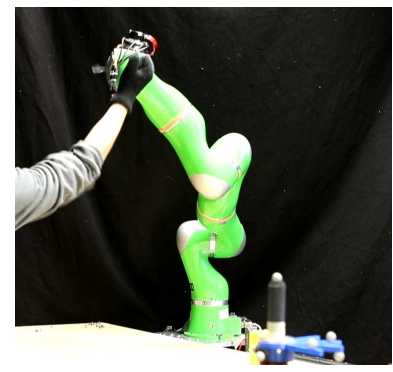

(a) A collision is exerted

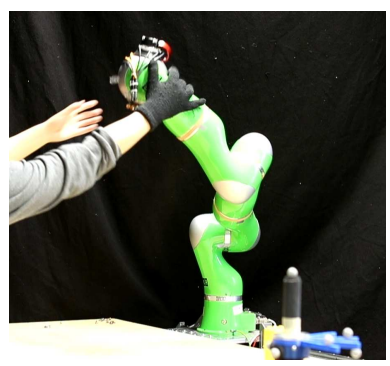

(c) An interaction is exerted.

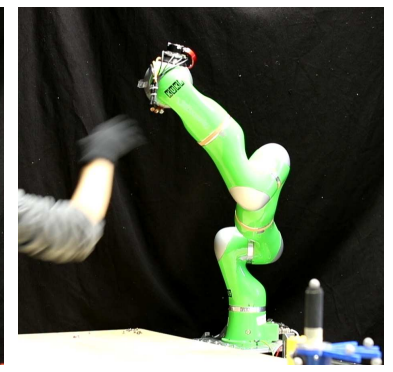

(b) An emergency stop.

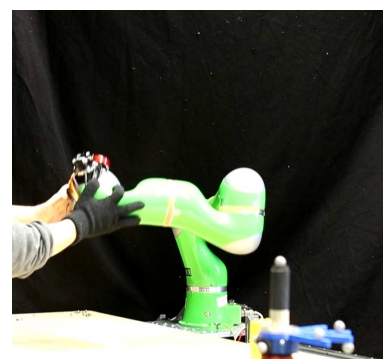

(d) The guidance mode
Fig. 10. The application demonstration of the online CDI diagnoser to the KUKA manipulator platform. The CDI scheme identifies collisions and interactions, such that different reaction routines are activated.

During the operation of the robot, the CDI scheme correctly detects and identifies most of the pHRI and enables the corresponding reaction procedure, although some week contacts and collisions fail to be detected. When the robot is smoothly moving, the false diagnosis of collisions and contacts is hardly witnessed. Nevertheless, a false diagnosis occurs when the robot motion shows large vibrations due to fierce motion or bad controller design. Therefore, a smooth functioning condition is still necessary. As for the responsiveness, the diagnosis delay is basically not obviously perceivable experimenters, which is acceptable in a typical human-robot collaborative task. Therefore, the demonstration confirms the performance of the proposed CDI and indicates its applicability to humanrobot collaboration tasks in practice.

\section{CONCLUSION}

In this paper, we develop a novel online CDI scheme for robot manipulators using supervised learning and the Bayesian decision theory. During the data collection process, we try to cover various execution conditions of the robot to ensure a representative data set. After carefully investigating the properties of the pHRI signals, we extract and select the features by analyzing their dependence and importance. As a result, the signal classifier presents excellent predictability and generalization ability. Based on this, we propose a fast 
and simply implementable online diagnosor with a quantified confidence index to depict the reliability of the diagnosis result based on the Bayesian decision theory. Examined by both theoretical analysis and experimental validation, the proposed CDI scheme shows a promising value to improve the safety of human-robot collaboration systems.

In general, our work in this paper has achieved the target raised in the beginning, i.e., to rapidly and correctly detect and identify collisions in their early stages. As a result, a collision is predicted before it vanishes, such that further damages or injuries can be avoided. The common ground of our work and the probabilistic-series-model-based methods is to make a decision using the observation series. Nevertheless, the proposed CDI scheme in this paper is more suitable to be widely applied in practice since it does not require prior knowledge of the signal dependence and assumptions on the data distribution.

It should be noted that the development of the CDI scheme, including the procedures of the data collection, feature engineering, and the model training, is conducted on a specific robot platform. Thus, the applicability of the developed CDI scheme to different platforms needs further investigations, which motivates our future work on the adaptation of the CDI scheme to a wider range of platforms. Another interesting topic is to compensate the effects of the varying robot loads, which can be solved by dynamic-model-based disturbance estimation methods [9]. To investigate the influence of the individual uncertainties on the classification accuracy, we will also collect collision data with more experiment subjects in future work.

\section{REFERENCES}

[1] D. Althoff, J. J. Kuffner, D. Wollherr, and M. Buss, "Safety assessment of robot trajectories for navigation in uncertain and dynamic environments," Autonomous Robots, vol. 32, no. 3, pp. 285-302, Apr 2012.

[2] A. M. Zanchettin, N. M. Ceriani, P. Rocco, H. Ding, and B. Matthias, "Safety in human-robot collaborative manufacturing environments: Metrics and control," IEEE Trans. Autom. Sci. Eng., vol. 13, no. 2, pp. 882-893, Apr 2016.

[3] S. Haddadin, A. Albu-Schäffer, and G. Hirzinger, "Safe physical humanrobot interaction: measurements, analysis and new insights," in Robotics research. Springer, 2010, pp. 395-407.

[4] P. A. Lasota, T. Fong, J. A. Shah et al., "A survey of methods for safe human-robot interaction," Foundations and Trends® in Robotics, vol. 5, no. 4, pp. 261-349, 2017.

[5] S. Haddadin, A. De Luca, and A. Albu-Schäffer, "Robot collisions: A survey on detection, isolation, and identification," IEEE Trans. Robot., vol. 33, no. 6, pp. 1292-1312, Dec 2017.

[6] M. Kimmel and S. Hirche, "Active safety control for dynamic humanrobot interaction," in Proc. IEEE Intell. Robot. Syst., Hamburg, Germany, Sept 2015, pp. 4685-4691.

[7] M. S. Wiig, K. Y. Pettersen, and T. R. Krogstad, "Collision avoidance for underactuated marine vehicles using the constant avoidance angle algorithm," IEEE Transactions on Control Systems Technology, 2019.

[8] Y. Sun, Z. Zhang, M. Leibold, R. Hayat, D. Wollherr, and M. Buss, "Protective control for robot manipulator by sliding mode based disturbance reconstruction approach," in Proc. IEEE Int. Conf. Advanced Intell. Mech., Munich, Germany, July 2017, pp. 1015-1022.

[9] Z. Zhang, M. Leibold, and D. Wollherr, "Integral sliding-mode observerbased disturbance estimation for euler-lagrangian systems," IEEE Transactions on Control Systems Technology, 2019.

[10] D. Kulić and E. Croft, "Pre-collision safety strategies for human-robot interaction," Autonomous Robots, vol. 22, no. 2, pp. 149-164, 2007.

[11] A. Kouris, F. Dimeas, and N. Aspragathos, "A frequency domain approach for contact type distinction in human-robot collaboration," IEEE Robot. Autom. Lett., vol. 3, no. 2, pp. 720-727, Jan 2018.
[12] G. Hu, C. Makkar, and W. E. Dixon, "Energy-based nonlinear control of underactuated euler-lagrange systems subject to impacts," IEEE Trans. Autom. Control, vol. 52, no. 9, pp. 1742-1748, Sept 2007.

[13] S. Haddadin, A. Albu-Schaffer, A. De Luca, and G. Hirzinger, "Collision detection and reaction: A contribution to safe physical human-robot interaction," in Proc. IEEE Int. Conf. Robot. Syst., Nice, France, Sept 2008, pp. 3356-3363.

[14] T. G. Puranik and D. N. Mavris, "Identification of instantaneous anomalies in general aviation operations using energy metrics," Journal of Aerospace Information Systems, pp. 1-17, 2019.

[15] L. Wellhausen, R. Ranftl, and M. Hutter, "Safe robot navigation via multi-modal anomaly detection," IEEE Robotics and Automation Letters, vol. 5, no. 2, pp. 1326-1333, 2020.

[16] S. W. Yahaya, A. Lotfi, and M. Mahmud, "A framework for anomaly detection in activities of daily living using an assistive robot," 2019.

[17] R. Isermann and P. Balle, "Trends in the application of model-based fault detection and diagnosis of technical processes," Control engineering practice, vol. 5, no. 5, pp. 709-719, May 1997.

[18] M. Geravand, F. Flacco, and A. De Luca, "Human-robot physical interaction and collaboration using an industrial robot with a closed control architecture," in Proc. IEEE Int. Conf. Robot. Autom., Karlsruhe, Germany, May 2013, pp. 4000-4007.

[19] B. Schuller, S. Steidl, A. Batliner, E. Bergelson, J. Krajewski, C. Janott, A. Amatuni, M. Casillas, A. Seidl, M. Soderstrom et al., "The interspeech 2017 computational paralinguistics challenge: Addressee, cold \& snoring," in ComParE, Interspeech 2017, 2017, pp. 3442-3446.

[20] K. Qian, C. Janott, Z. Zhang, D. Jun, A. Baird, C. Heiser, W. Hohenhorst, M. Herzog, W. Hemmert, and B. Schuller, "Teaching machines on snoring: a benchmark on computer audition for snore sound excitation localisation," Archives of Acoustics, vol. 43, no. 3, pp. 465-475, 2018.

[21] K. Qian, Z. Zhang, A. Baird, and B. Schuller, "Active learning for bird sound classification via a kernel-based extreme learning machine," The Journal of the Acoustical Society of America, vol. 142, no. 4, pp. 17961804, Sept 2017.

[22] R. Yan, R. X. Gao, and X. Chen, "Wavelets for fault diagnosis of rotary machines: A review with applications," Signal processing, vol. 96, pp 1-15, Mar 2014.

[23] D. Verstraete, A. Ferrada, E. L. Droguett, V. Meruane, and M. Modarres, "Deep learning enabled fault diagnosis using time-frequency image analysis of rolling element bearings," Shock and Vibration, 2017.

[24] A. Rahimi, K. D. Kumar, and H. Alighanbari, "Fault detection and isolation of control moment gyros for satellite attitude control subsystem,' Mechanical Systems and Signal Processing, vol. 135, p. 106419, 2020

[25] F. Karim, S. Majumdar, H. Darabi, and S. Chen, "Lstm fully convolutional networks for time series classification," IEEE Access, vol. 6, pp. 1662-1669, 2017.

[26] S.-Y. Shao, W.-J. Sun, R.-Q. Yan, P. Wang, and R. X. Gao, "A deep learning approach for fault diagnosis of induction motors in manufacturing," Chin. J. Mech. Eng., vol. 30, no. 6, pp. 1347-1356, Oct 2017.

[27] H. Wu, D. Liu, S. Duan, Y. Guan, and J. Rojas, "Multimodal sparse representation for anomaly classification in a robot introspection system," in 2018 IEEE International Conference on Robotics and Biomimetics (ROBIO). IEEE, 2018, pp. 1594-1600.

[28] I. Agriomallos, S. Doltsinis, I. Mitsioni, and Z. Doulgeri, "Slippage detection generalizing to grasping of unknown objects using machine learning with novel features," IEEE Robot. Autom. Lett., vol. 3, no. 2, pp. 942-948, Apr 2018.

[29] A.-N. Sharkawy, P. N. Koustoumpardis, and N. A. Aspragathos, "Manipulator collision detection and collided link identification based on neural networks," in International Conference on Robotics in Alpe-Adria Danube Region. Springer, 2018, pp. 3-12.

[30] K. Narukawa, T. Yoshiike, K. Tanaka, and M. Kuroda, "Real-time collision detection based on one class svm for safe movement of humanoid robot," in Proc. IEEE Humanoid Robotics, Birmingham, United Kindom, Nov 2017, pp. 791-796.

[31] S. Golz, C. Osendorfer, and S. Haddadin, "Using tactile sensation for learning contact knowledge: Discriminate collision from physical interaction," in 2015 IEEE International Conference on Robotics and Automation (ICRA), May 2015, pp. 3788-3794.

[32] S. Luo, H. Wu, H. Lin, S. Duan, Y. Guan, and J. Rojas, "Fast, robust, and versatile event detection through hmm belief state gradient measures," in 2018 27th IEEE International Symposium on Robot and Human Interactive Communication (RO-MAN). IEEE, 2018, pp. 1-8.

[33] D. Romeres, D. K. Jha, W. Yerazunis, D. Nikovski, and H. A. Dau, "Anomaly detection for insertion tasks in robotic assembly using gaussian process models," in 2019 18th European Control Conference (ECC). IEEE, 2019, pp. 1017-1022. 
[34] E. Di Lello, M. Klotzbücher, T. De Laet, and H. Bruyninckx, "Bayesian time-series models for continuous fault detection and recognition in industrial robotic tasks," in 2013 IEEE/RSJ International Conference on Intelligent Robots and Systems. IEEE, 2013, pp. 5827-5833.

[35] D. Park, H. Kim, Y. Hoshi, Z. Erickson, A. Kapusta, and C. C. Kemp, "A multimodal execution monitor with anomaly classification for robot-assisted feeding," in 2017 IEEE/RSJ International Conference on Intelligent Robots and Systems (IROS). IEEE, 2017, pp. 5406-5413.

[36] J. Rojas, S. Luo, D. Zhu, Y. Du, H. Lin, Z. Huang, W. Kuang, and K. Harada, "Online robot introspection via wrench-based action grammars," in Proc. IEEE Intell. Robot. Syst., Vancouver, Canada, Sept 2017, pp. 5429-5436.

[37] R. Bischoff, J. Kurth, G. Schreiber, R. Koeppe, A. Albu-Schäffer, A. Beyer, O. Eiberger, S. Haddadin, A. Stemmer, G. Grunwald et al., "The kuka-dlr lightweight robot arm-a new reference platform for robotics research and manufacturing," in ISR 2010 (41st international symposium on robotics) and ROBOTIK 2010 (6th German conference on robotics). VDE, 2010, pp. 1-8.

[38] K. Qian, C. Janott, Z. Zhang, C. Heiser, and B. Schuller, "Wavelet features for classification of vote snore sounds," in Proc. IEEE Int. Conf. ICASSP., Shanghai, China, Mar 2016, pp. 221-225.

[39] K. Qian, C. Janott, V. Pandit, Z. Zhang, C. Heiser, W. Hohenhorst, M. Herzog, W. Hemmert, and B. Schuller, "Classification of the excitation location of snore sounds in the upper airway by acoustic multifeature analysis," IEEE Trans. Biomed. Eng., vol. 64, no. 8, pp. 1731-1741, Oct 2017

[40] M. D. Prieto, G. Cirrincione, A. G. Espinosa, J. A. Ortega, and H. Henao, "Bearing fault detection by a novel condition-monitoring scheme based on statistical-time features and neural networks," IEEE Trans. Ind. Electron., vol. 60, no. 8, pp. 3398-3407, Aug 2013.

[41] S. Mazilu, M. Hardegger, Z. Zhu, D. Roggen, G. Troster, M. Plotnik, and J. M. Hausdorff, "Online detection of freezing of gait with smartphones and machine learning techniques," in Proc. IEEE PervasiveHealth, San Diego, California USA, May 2012, pp. 123-130.

[42] K. Qian, C. Janott, J. Deng, C. Heiser, W. Hohenhorst, M. Herzog, N. Cummins, and B. Schuller, "Snore sound recognition: on wavelets and classifiers from deep nets to kernels," in Conf Proc IEEE Eng. Med. Biol. Soc., Jeju Island, Korea, July 2017, pp. 3737-3740.

[43] J. M. Taylor, "Kendall's and spearman's correlation coefficients in the presence of a blocking variable," Biometrics, pp. 409-416, 1987.

[44] K. Kira, L. A. Rendell et al., "The feature selection problem: Traditional methods and a new algorithm," in Aaai, vol. 2, 1992, pp. 129-134.

[45] I. Kononenko, E. Šimec, and M. Robnik-Šikonja, "Overcoming the myopia of inductive learning algorithms with relieff," Applied Intelligence, vol. 7, no. 1, pp. 39-55, 1997.

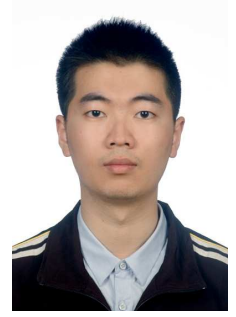

Zengiie Zhang (S'19) received his Bachelor and Master degrees from Harbin Institute of Technology, China, in 2013 and 2015 respectively. He is currently a Research Associate at the Chair of Automatic Control Engineering of the Technical University of Munich, Germany, while pursuing the doctoral degree. His research interests include sliding mode control, fault detection and isolation and humanrobot collaboration.

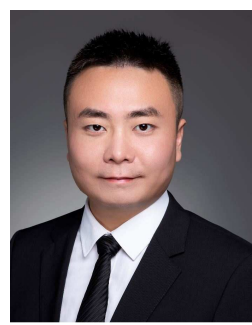

Kun Qian (S'14-M'19) received his doctoral degree (Dr.-Ing.) for his study on automatic general audio signal classification in 2018 in electrical engineering and information technology from Technische Universität München (TUM), Germany. Currently, he is working as a JSPS Postdoctoral Research Fellow in the Educational Physiology Laboratory, Graduate School of Education, The University of Tokyo (UTokyo), Japan. He was sponsored by fellowships to be the visiting researcher at the Nanyang Technological University (NTU), Singapore, the Tokyo Institute of Technology (Tokyo Tech), Japan, and the Carnegie Mellon University (CMU), USA. His main research interests include signal processing, machine learning, biomedical engineering, and deep learning.

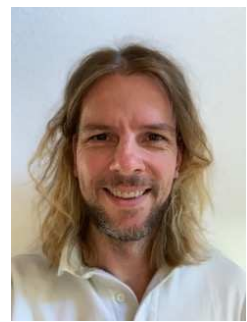

Björn W. Schuller (M'06-SM'15-F'18) received his diploma in 1999, his doctoral degree for his study on automatic speech and emotion recognition in 2006, and his habilitation and Adjunct Teaching Professorship in the subject area of signal processing and machine intelligence in 2012, all in electrical engineering and information technology from Technische Universität München (TUM), Germany. He is a tenured Full Professor heading the Chair of Embedded Intelligence for Health Care and Wellbeing, University of Augsburg, Germany, and a Reader (Associate Professor) in Machine Learning heading GLAM - the Group on Language, Audio \& Music, Department of Computing at the Imperial College London in London, UK. Dr. Schuller is elected member of the IEEE Speech and Language Processing Technical Committee, Editor in Chief of the IEEE Transactions on Affective Computing, President-emeritus of the AAAC, Fellow of the IEEE, and Senior Member of the ACM. He (co-)authored 5 books and more than 700 publications in peer reviewed books, journals, and conference proceedings leading to more than 28000 citations (h-index 79).

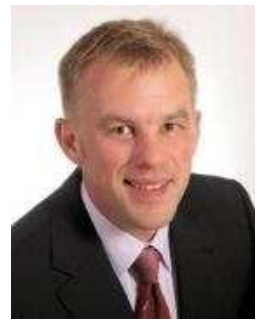

Dirk Wollherr (S'01-M'11-SM'11) received the Diplom-Ingenieur degree and the Doctor of Engineering degree, both in electrical engineering, and the Habilitation degree from Technical University Munich, Germany, in 2000, 2005, and 2013, respectively. From 2001 to 2004, he was a Research Assistant with the Control Systems Group, Technische Universität Berlin, Germany. In 2004, he was with the Yoshihiko Nakamura Laboratory, The University of Tokyo, Japan. Since 2014, he has been a Professor with the Chair of Automatic Control Engineering, Department of Electrical and Computer Engineering, Technical University Munich. His research interests include automatic control, robotics, autonomous mobile robots, human-robot interaction, and humanoid walking. 